\title{
Étude archéologique de la plate-forme du Mālamatagata à 'Utuleve, île de 'Uvea (Wallis)
}

\section{Maurice Hardy}

\section{(2) OpenEdition}

1 Journals

Édition électronique

URL : http://journals.openedition.org/jso/5851

DOI : $10.4000 /$ jso. 5851

ISSN : $1760-7256$

Éditeur

Société des océanistes

\section{Édition imprimée}

Date de publication : 30 juin 2009

Pagination : 69-76

ISBN : 978-2-85430-024-6

ISSN : 0300-953x

\section{Référence électronique}

Maurice Hardy, «Étude archéologique de la plate-forme du Mālamatagata à 'Utuleve, île de 'Uvea (Wallis) », Journal de la Société des Océanistes [En ligne], 128 | janvier-juin 2009, mis en ligne le 30 juin 2012, consulté le 02 mai 2019. URL : http://journals.openedition.org/jso/5851 ; DOI : 10.4000/ jso.5851

\section{(c) Tous droits réservés}




\section{Étude archéologique de la plate-forme du Mālamatagata à 'Utuleve, île de 'Uvea (Wallis)}

par

Maurice HARDY

\section{RÉSUMÉ}

La tradition orale rapporte que la plate-forme du Mālamatagata, sur le site de 'Utuleve à 'Uvea (Wallis), a été construite par un chef de Tonga à l'occasion de l'accouchement de sa fille, afin de montrer à tous le haut rang qu'elle occupait dans la société tongienne. Au début des années 1990, les autorités du territoire ont souhaité que soit réalisée l'étude scientifique et la mise en valeur de cette structure. Ce programme a permis de montrer que le monument avait été construit en plusieurs étapes et a contribué à mieux définir les techniques de construction employées pour l'édification des monuments anciens en Polynésie occidentale.

Mots-CLÉS : archéologie, 'Uvea, Wallis, restauration, Tongiens

C'est sur les conseils de José Garanger et avec son appui que j'ai intégré, en 1993, le programme de recherches dirigé par Daniel Frimigacci sur l'archéologie de Wallis, afin d'aider aux travaux de restauration de monuments anciens et aux fouilles. Les travaux du Professeur José Garanger dans la presqu'île de Tautira à Tahiti ainsi que la fouille du marae Ta'ata et surtout, celles menées à Vanuatu (ex-Nouvelles-Hébrides), ont très fortement inspiré mes travaux à Wallis. C'est en effet lui qui mena au plus loin l'utilisation des

\section{ABSTRACT}

The oral traditions report that the Mãlamatagata platform, located on the site of 'Utuleve on 'Uvea island (Wallis), was constructed by a chief from Tonga, as a birthplace for his grand-son, in order to show to all the high rank occupied by his daughter in the Tongan society. In the early $1990^{s}$, the political authorities of the Territory decided to fulfil a scientific study and a restauration project on this monument. This program has shown that the monument had gone through various episodes of construction, and provided a better knowledge of the construction techniques used for the building of ancient monuments in Western Polynesia.

KEYwORDS: archaeology, East Uvea, Wallis, restauration, Tongans

traditions orales en archéologie en y voyant de véritables «documents historiques». Avec Daniel Frimigacci, nous avons restauré plusieurs monuments tongiens dans la partie sud de l'île, là où des groupes venus de Tonga s'installèrent au cours du deuxième millénaire après J.-C. Ils construisirent à 'Uvea d'imposants bâtiments qu'ils modifièrent et remanièrent jusqu'à très récemment. Les travaux archéologiques ont aidé à la compréhension de l'architecture de ces structures et des phases successives de construction

* UMR CNRS 7041 ArScAn, maurice.hardy@mae.u-paris10.fr. 


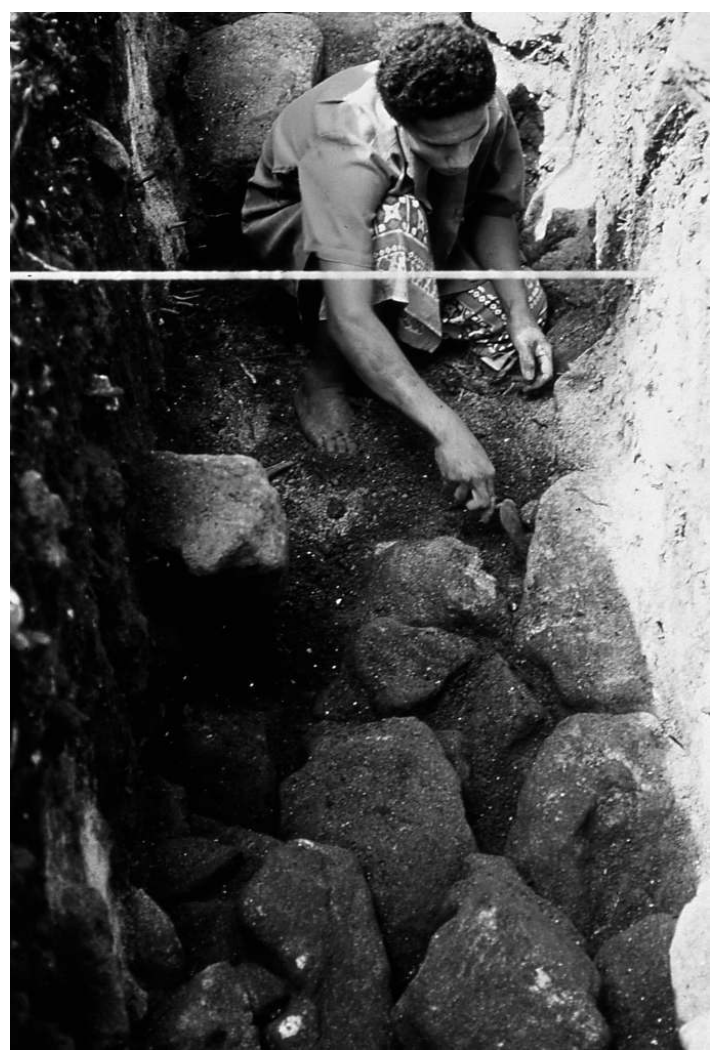

Pното 1. - Fouille à 'Utuleve (cliché 4, CTRDP, 1995)

(photo 1). Les restaurations, quant à elles, ont permis de concrètement restituer aux Wallisiens une partie des informations collectées sur le patrimoine culturel de l'île. En 1995, j'ai pris part aux travaux sur la plate-forme nommée Mālamatagata que le Service culturel de Wallis et les propriétaires (entre autres, Mikaele Tufele, membre du clan propriétaire terrien) voulaient voir restaurée (Frimigacci et Hardy, 1997).

Lorsqu'en 1767, le capitaine britannique Samuel Wallis approcha l'île de 'Uvea (qui porte aujourd'hui son nom), des Océaniens y vivaient déjà depuis plusieurs millénaires. 'Uvea se trouve dans une région considérée comme le berceau de la culture polynésienne, entourée par les îles Samoa à l'est, les îles Fidji et Futuna au sudouest et Tonga au sud (figure 1). 'Uvea, île volcanique peu élevée, ne comporte aucune rivière mais possède plusieurs lacs. Le premier peuplement humain, il y a environ trois mille ans, s'est fait lors de la progression austronésienne à travers le Pacifique sud-ouest, caractérisée par la production de poteries Lapita décorées. Aux vestiges archéologiques viennent s'ajouter les traditions orales qui renseignent sur l'histoire de Wal

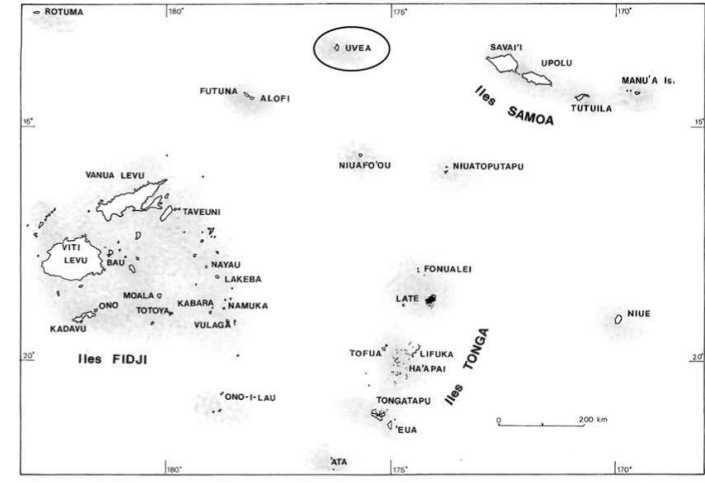

Figure 1. - 'Uvea et les îles voisines de Fidji et de Polynésie occidentale (Hardy, d'après carte IGN, série Bleue 1 :25000, IGN Paris, 1987)

lis au cours des derniers siècles avant l'arrivée des Européens (Henquel, 1910). D'après ces récits, l'île était occupée à cette époque par les Tongiens qui y avaient édifié des forts et des chemins fortifiés (Frimigacci et Vienne, 2001). Ces derniers avaient également instauré un système de chefferie à titres, de type pyramidal, qui s'est maintenu jusqu'à nos jours (Frimigacci et Vienne, 2006).

Cet article présente les données obtenues lors de l'étude menée sur la plate-forme du Mālamatagata. Après avoir résumé les informations de traditions orales dont nous disposons, les données archéologiques seront détaillées.

\section{Les origines du Mālamatagata}

D'après les traditions orales, la construction de la plate-forme du Mālamatagata aurait été liée à la présence tongienne sur 'Uvea. Au cours du partage de l'île entre les chefs Hoko, Fakate et Kalāfilia ${ }^{1}$, ce dernier se serait fait attribuer les terres situées à l'ouest du lac de Lanutavake jusqu'à Lauliki et Kaupalua, ainsi que les lacs Lanutuli et Lalolalo. Kalāfilia choisit de bâtir une résidence à 'Utuleve, sur la côte ouest de 'Uvea (Burrows, 1937 ; Frimigacci, Siorat et Vienne, 1982 ; Frimigacci et Vienne, 2006). C'est cet ensemble architectural, comprenant le Mālamatagata, que les responsables coutumiers et les élus du territoire nous ont demandé de restaurer. L'ensemble des travaux de fouilles et de restauration s'est étalé sur trois ans, à raison de trois mois par campagne.

Les récits sur le Mālamatagata apparaissent dans deux formes d'expression de la tradition 


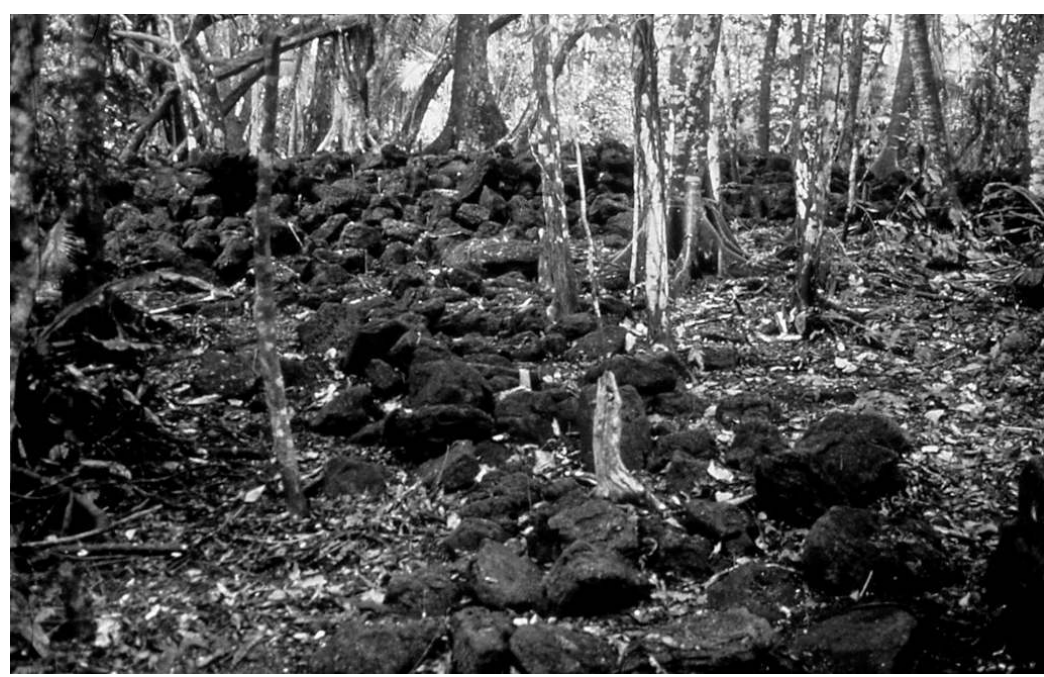

Рното 2. - Chemins des nobles (cliché 10, CTRDP, 1995)

orale (talatuku) à 'Uvea ${ }^{2}$. La première, constituée de danses (tapaki) ou de chants (ta'agalave), est formellement figée. La seconde, comprenant des textes oraux (talanoa, fagona, fakamatala), est plus libre. Ces derniers rapportent que Kalāfilia fit construire sa résidence à 'Utuleve. Plus tard, lorsque sa fille, Ohopulu, fut sur le point d'accoucher, elle demanda à son père de montrer à tous son appartenance à un haut lignage. Aussi fit-il construire une plate-forme près de sa résidence, sur laquelle eut lieu la naissance de son petit-fils Alokuaulu. C'est à la lueur de Tongiens décapités et transformés en torches, que se serait déroulé l'accouchement, d'où le nom de Mālamatagata (hommes-torches) donné au monument. Un chant ancien (ta'agalave) précise que le père de l'enfant aurait été Taimalelagi, frère du Tui Tonga. D'après ce chant, c'est Taimalelagi qui aurait donné l'ordre d'enterrer plusieurs hommes debout, dans des cavités aménagées sur la plate-forme (Burrows, 1937).

\section{Les données archéologiques}

La plate-forme du Mālamatagata est située à l'arrière du bord de mer de la plage de 'Utuleve, sur la côte ouest de 'Uvea, face aux passes d'Avatolu, de Fuga'Uvea et de Fatumanini (figure 2). La dune quaternaire positionnée en limite du marais de To'ogatoto renferme un site Lapita, dont la première occupation remonte à près de trois mille ans (Sand, 1998). La plate-forme du Mālamatagata est un monument de forme rectangulaire, d'environ $30 \mathrm{~m}$ sur $15 \mathrm{~m}$, prolongé

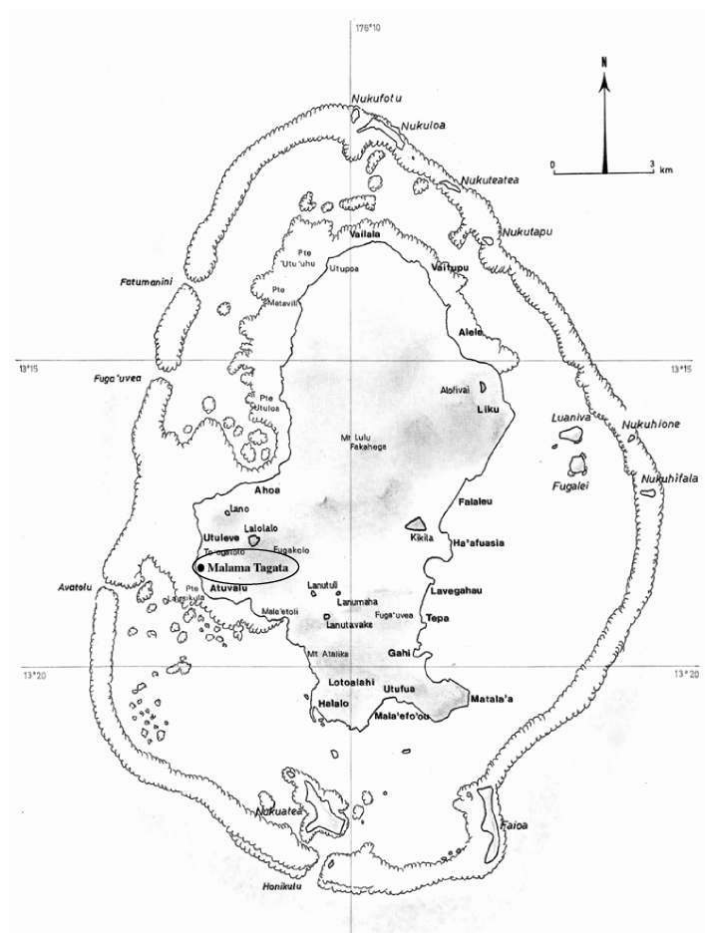

FiguRE 2. - Localisation du Mālamatagata dans l'île de Wallis

par une liaison basse empierrée, probablement une allée de type ala hou 'eiki (chemin des nobles, cf. photo 2), les nobles ne devant pas se déplacer au même niveau que les roturiers (Burrows, 1937 ; Frimigacci et Hardy, 1997). Cette allée, très bouleversée par les cultures, rejoint le marais To'ogatoto (voir photo 6) dans lequel se trouvent plusieurs structures dont un puits et des enceintes de pierres destinées, selon la tradition orale,

2. NDLR. - Voir ci-après la tradition orale Ko te mālama tagata. Les « hommes-torches », histoire de Kalāfilia, proposée par Claire Moyse-Faurie. 


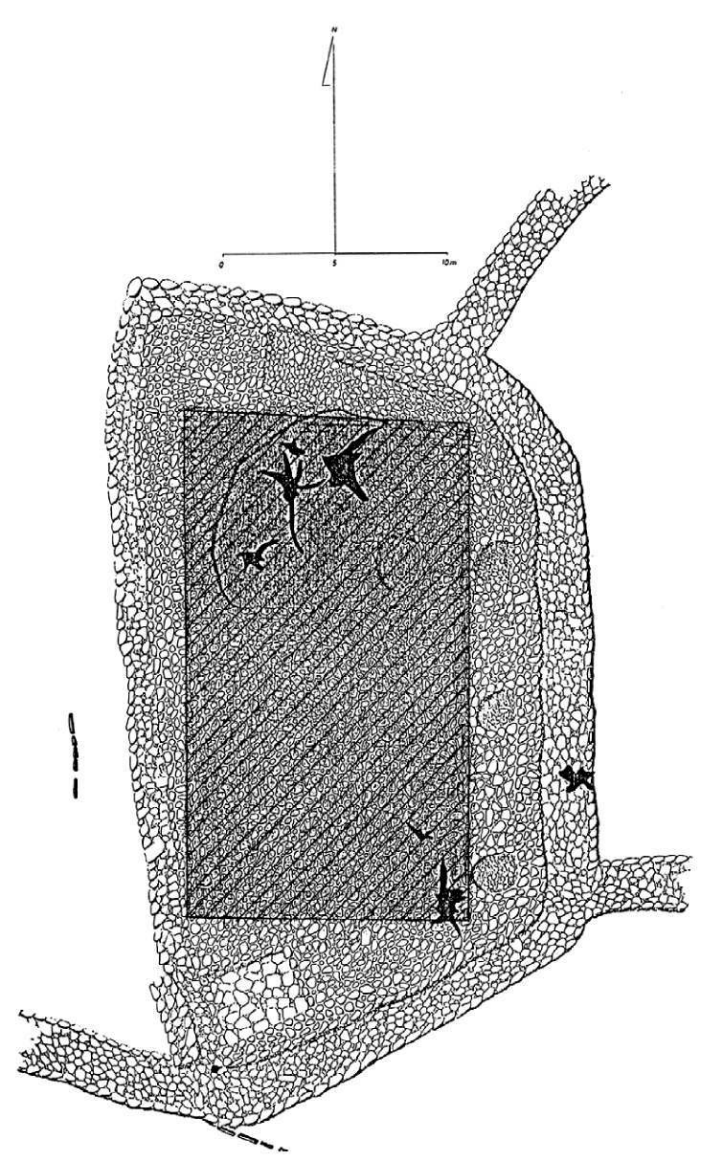

FIGURE 3. - Extension maximale du monument ancien vu lors de la fouille, sous le Mālamatagata (Hardy, 1996)

au bain des nobles. Une autre allée, au sud, relie la résidence du Kalāfilia au bord de mer en passant par la partie sud du monument. Au sudouest, une allée vient se raccorder en provenance du sud; elle s'enfonce dans la brousse et va rejoindre la route ancienne.

La plate-forme du Mālamatagata est une construction en pierres sèches, comprenant à l'est trois postes de guet et un mur. La surface de la plate-forme est horizontale, avec un ancien dallage, probablement réalisé pour égaliser la surface du monument. Elle comprend néanmoins au nord une cuvette sableuse et, à l'est, une surface corallienne. Plusieurs squelettes avaient été enterrés dans une cuvette creusée dans la partie ouest. Ces squelettes, placés en position allongée sur un pavage grossier, étaient fortement perturbés par les racines des arbres et les éboulis. Un mur circonscrit l'ensemble.

\section{La structure ancienne}

Les sondages réalisés sur et en bordure du monument, ont montré au moins deux phases dans la construction du Mālamatagata. La partie supérieure de la stratigraphie est constituée de blocs empilés. À environ $90 \mathrm{~cm}$ sous la surface du dallage, un mélange compact de terre et de pierres d'environ $30-40 \mathrm{~cm}$ d'épaisseur est présent, vestige d'une structure plus ancienne dont la construction remonte probablement aux années 1300 de notre ère (Frimigacci et Vienne, 2001, 2006). Les vestiges appartenant à cette construction ancienne, présents sous une bonne part de la structure plus récente, sont difficiles à analyser car ils sont masqués par les aménagements postérieurs de la structure (figure 3). La forme exacte de la première plate-forme est donc impossible à déterminer. Le monument initial était construit à l'aide de blocs de corail mélangés à des sédiments divers : du corail, un sédiment sablo-argileux, des galets de plage et des graviers (figure 4). Les bâtisseurs ont utilisé en remblai un sol ancien prélevé à l'ouest de la structure, contenant un grand nombre de tessons de poterie de type 'Utuleve III. Cet événement se serait déroulé aux alentours de 1300 de notre ère, ce qui correspondrait au règne de Puhi, roi mythique enterré à Atuvalu (Frimigaci et Vienne, 2001, 2006). Le niveau de remblai a recouvert un foyer de $80 \mathrm{~cm}$ de diamètre pour $30 \mathrm{~cm}$ de profondeur maximale. La sole est parsemée de plaques de terre brûlée, séparées les unes des autres par une fine couche de terre, indiquant plusieurs utilisations. Une ébauche d'herminette (figure 5) et une herminette polie ont été mises au jour en surface du foyer et, presque au fond, un éclat d'herminette polie se trouvait parmi d'autres éclats thermiques.

Deux datations sur charbons ont été réalisées sur cet horizon ancien de construction. La première, issue du niveau de remblai, a donné un résultat de 1363 cal AD (ANU 9451). Des charbons prélevés dans le foyer sous le remblai ancien du Mālamatagata, ont donné un résultat de 1247 cal AD (ANU 10071).

\section{La structure de surface}

Le nettoyage du couvert végétal a dégagé la plate-forme (photos 3-4 et figure 6) et fait apparaître, par endroits, les assises régulières parfaitement conservées du monument, composées de blocs de basalte bien appareillés. La plate-forme supérieure du monument est formée d'un dallage et parfois d'un pavage. Le dallage était composé d'un assemblage de grandes dalles ayant plus de $40-50 \mathrm{~cm}$ de grand axe. Le pavage, quant à lui, était constitué d'un assemblage de pierres plates (mesurant entre 20 et $35 \mathrm{~cm}$ de grand axe), dis- 


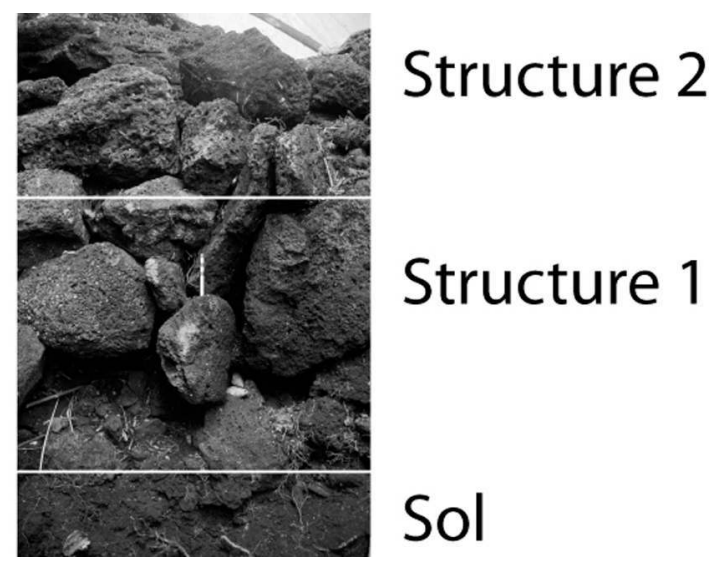

FIgURE 4. - Coupe, effectuée dans le Mālamatagata, montrant les deux types de construction
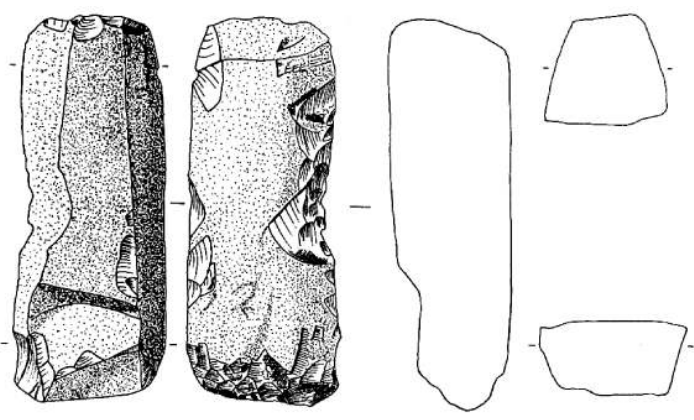

3 an

FiguRE 5. - Dessin d'une ébauche d'herminette mise au jour lors des fouilles

posées de façon à former un sol. Au pied des murs, se distinguaient les vestiges de ce qui devait etre un chemin assurant une circulation autour du monument, réalisé avec des pierres de petit module (entre 20 et $35 \mathrm{~cm}$ de grand axe) et présentant un tassement qui pourrait être le résultat du piétinement. Le reste du pavage est très bouleversé par les racines des arbres et les animaux. Un peu plus à l'est, une petite cuvette est remplie de corail très corrodé. Une grande cuvette de sable, bordée par endroits de pierres plantées de chant ou d'assises de pierres formant une sorte de muret, est présente au centre du Mālamatagata. Sa fouille a mis au jour un squelette enterré dans la partie nord de cette dépression, qui semble avoir été déposé dans un espace compartimenté par des dalles puis recouvert de sable. De nombreux fragments d'os humains, ainsi que des os longs en fagot, ont été observés à la base de quatre grands arbres : un ma'ota (Pongamia pinnata (L.) Merr., Légumineuses) et trois poumuli (Flueggea flexuosa Muell., Euphorbiacées) qui poussent aujourd'hui sur le monument. Les poumuli, souvent retrouvés sur les lieux sacrés, servaient de poteaux de torture.

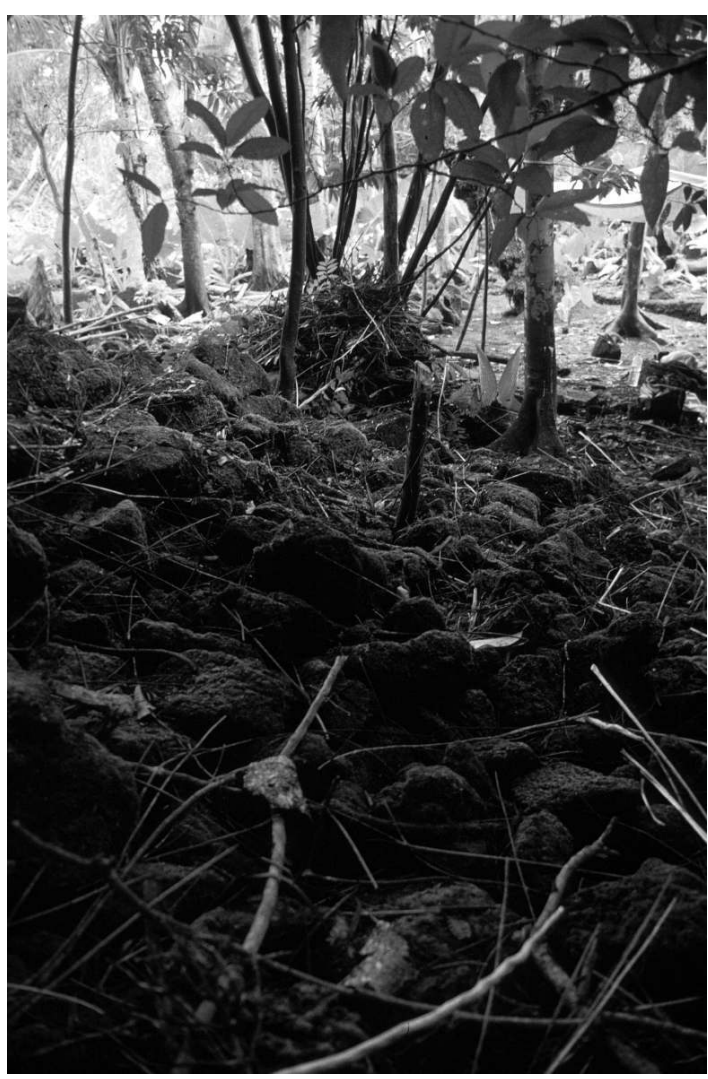

Рното 3. - Entrée nord du Mālamatagata avant restauration (cliché de l'auteur)

La végétation, le passage des hommes et les cultures de kape ont complètement bouleversé le pavage et les murs.

Pour mener à bien la restauration du monument, nous avons démantelé les murs existants et démonté le dallage supérieur. Ensuite, la reconstruction a commencé par les murs éboulés, en utilisant les grandes dalles présentes dans les éboulis, à l'image de ce qui existait encore dans les pans de murs en place, en positionnant ces dalles de façon à ce que leur face la plus plane apparaisse en façade. Le pavage supérieur a été restauré en liaison avec le sommet des murs. Le dégagement du monument a permis d'étudier, le long du mur est, trois dépressions présentant un espacement à peu près régulier, construit au-delà des limites du monument le plus ancien. Ces dépressions, profondes d'environ $70 \mathrm{~cm}$, correspondent sans doute à des postes de guet qui permettaient à des guerriers de surveiller sans être vus, protégés par le mur du Mālamatagata. Le même type de construction a déjà été noté sur la résidence du Talietumu, plus particulièrement sur le mur de protection formant le fort de Kolonui. Le fond de chaque dépression est pavé, les murs sont construits en blocs basaltiques empilés ou à l'aide de pierres disposées de chant dans le mur. 


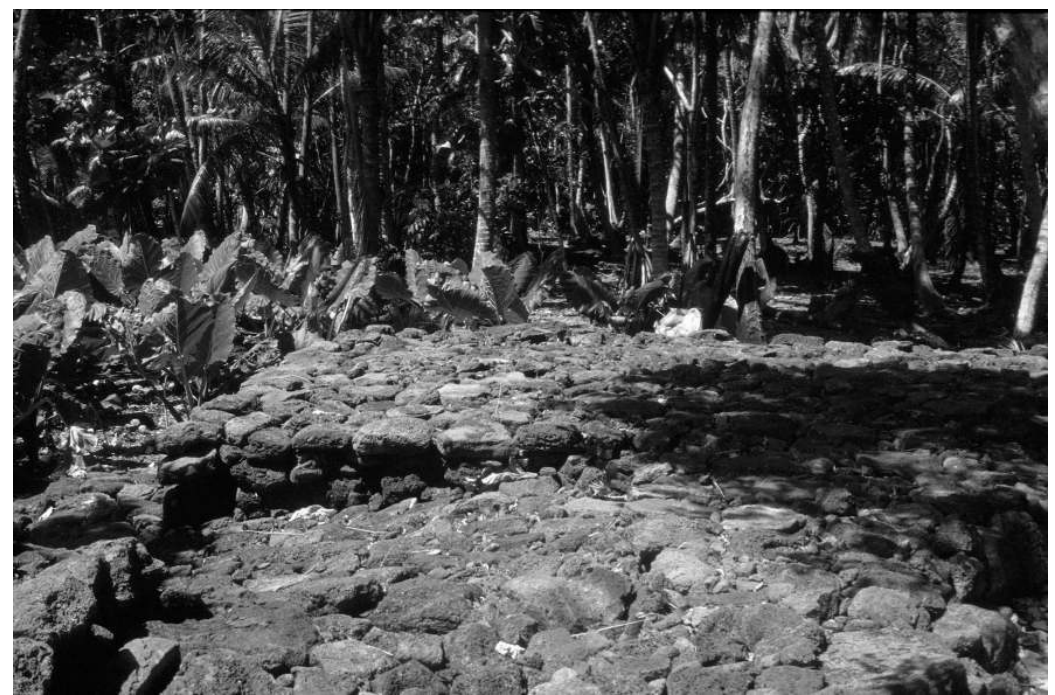

Рното 4. - Entrée nord du Mālamatagata après restauration (cliché de l'auteur)

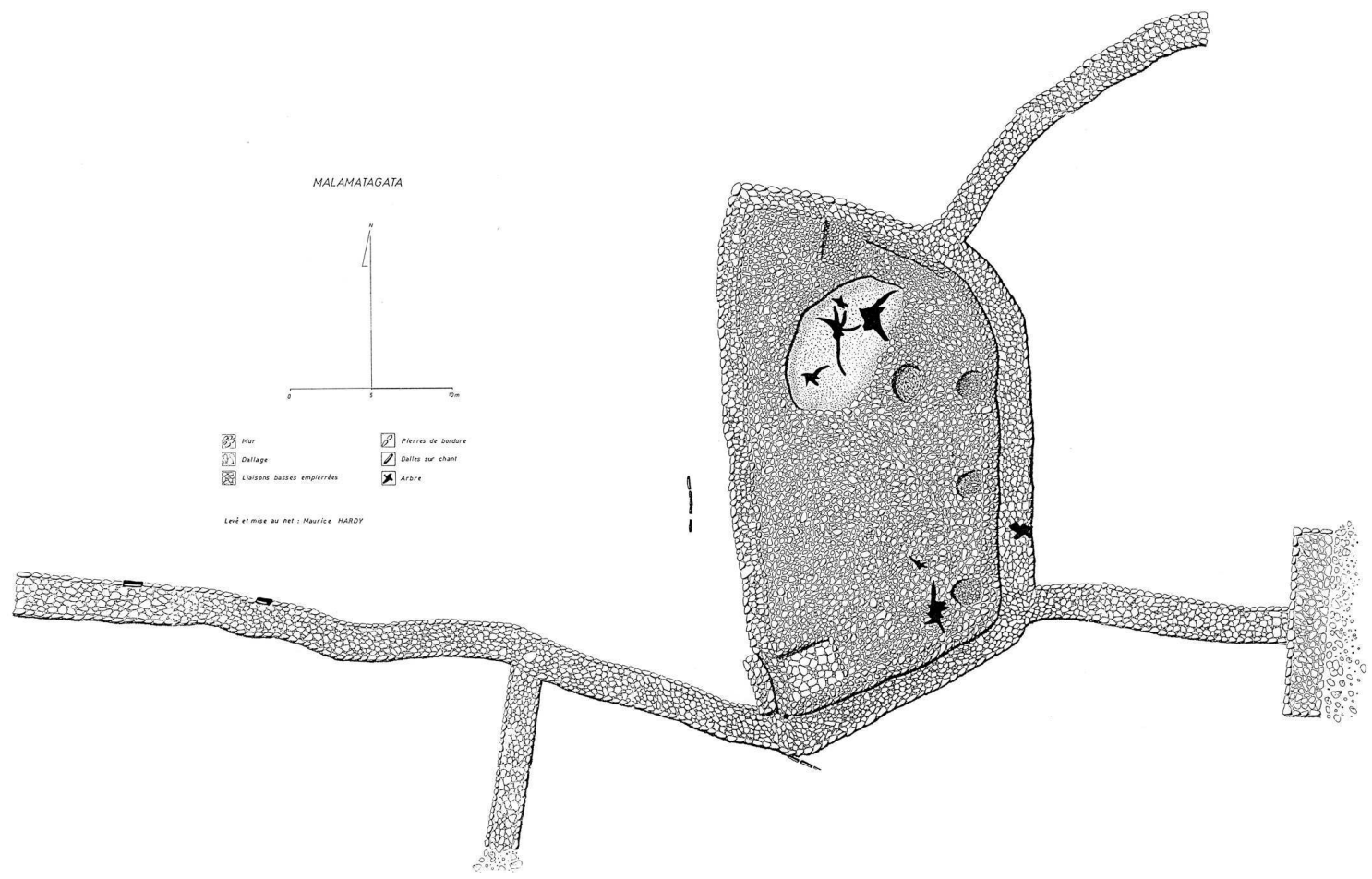

Figure 6. - Plan du Mālamatagata après restauration (levé et mise au net par l'auteur)

Les murs du monument sont constitués de blocs de basalte empilés les uns sur les autres. Ils ne présentent pas de traces d'appareillage particulier. Les constructeurs ont toutefois positionné les dalles de façon à ce que leur surface plane se présente en façade du mur, donnant ainsi l'aspect d'une construction relativement homogène, aux assises régulières. De place en place, les assises sont retenues par des clefs, constituées de pierres souvent carrées servant de coins. Ni les murs ni les angles ne présentent de chaînage, bien que certains angles aient des pierres d'angle. La rugosité naturelle des blocs de basalte fait que les pierres s'accrochent entre elles, réduisant les risques d'éboulements.

Les fondations du monument sont constituées de grandes dalles posées à plat sur le sol.

Les liaisons basses empierrées se présentent sous la forme d'allées. Elles forment un réseau ceinturant le monument, à la manière d'un rond- 


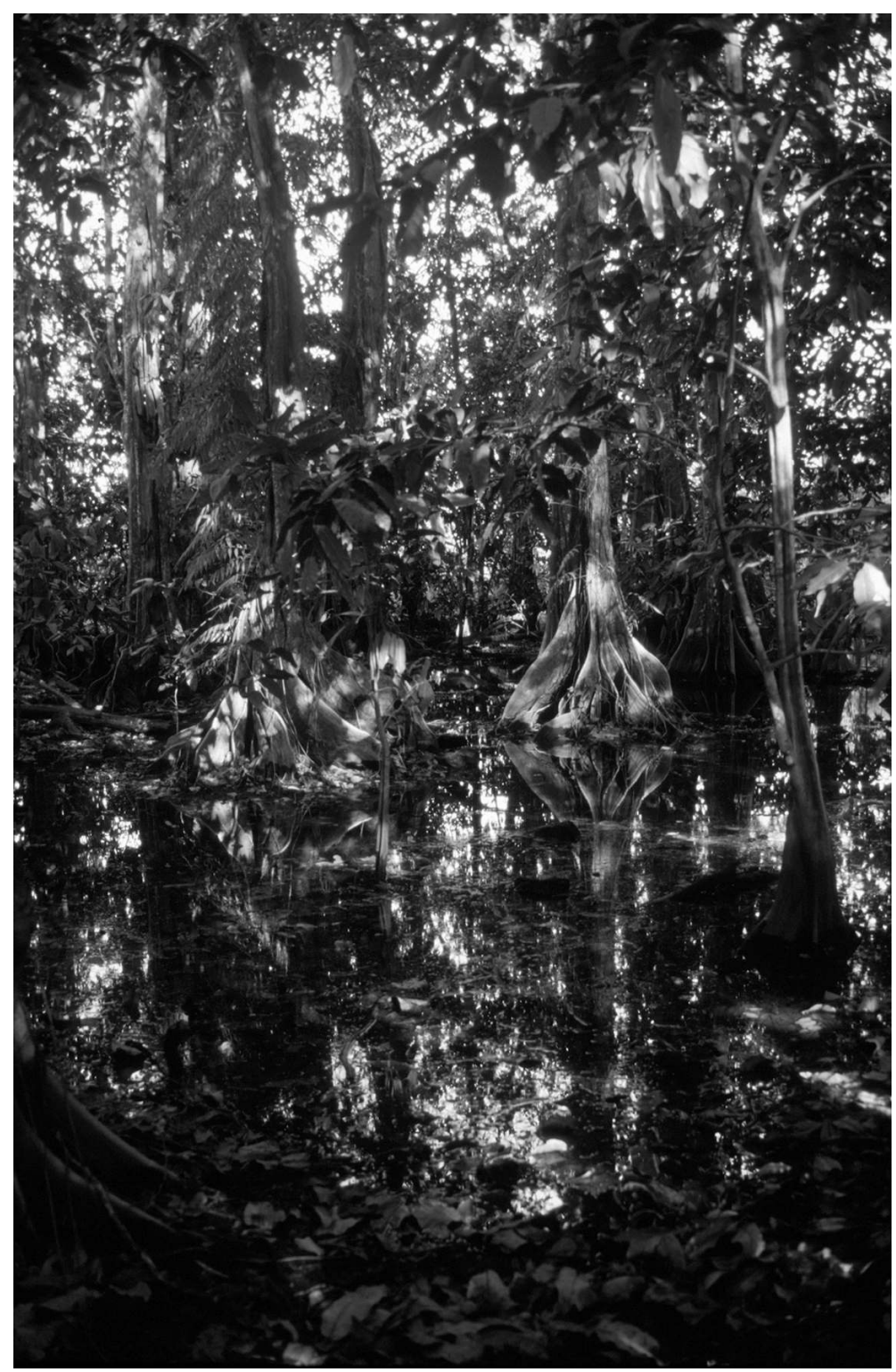

Рното 5. - Vue du marais To'ogatoto (cliché de l'auteur)

point permettant d'emprunter les entrées nord ou sud-ouest du Mālamatagata. Elles laissaient aux nobles la possibilité de se déplacer dans un espace organisé, tout en étant au-dessus des serviteurs, d'où le nom de ala hou 'eiki qui leur est donné en tongien, qui peut être traduit par « chemin des nobles » (ala hou aliki en wallisien). Les entrées de la structure forment un plan incliné, sans degrés (photo 4). L'entrée du sud-ouest est protégée par une chicane, comme c'est l'usage dans les monuments de cette époque. Ces allées sont construites à l'aide de blocs de basalte posés directement sur le sol, sans aucune fondation. Les bordures de ces liaisons sont très légèrement enfouies, mais elles sont parfois constituées par des dalles plantées de chant dans le sol. Entre les bordures, le remplissage se compose de blocs de basalte relativement plats et de blocaille (cailloutis comblant les interstices).

Ces voies de communication pour nobles nous renseignent sur les relations du monument avec son environnement. On peut ainsi noter que le Mālamatagata est relié à la résidence de Kalāfilia, toute proche. Il a vraisemblablement servi de place de réunion (mala'e) pour les chefs résidant sur le Kalāfilia. Une de ces liaisons basses empierrées se dirige tout droit vers le marais To'ogatoto (marais de sang) dans lequel on a retrouvé des traces de structures en basalte. La tradition rapporte que se trouvaient là les bains 
des nobles. Les membres âgés de la famille royale se rappellent encore être allés aux bains à cet endroit avec leurs parents.

Le Mālamatagata est également en liaison avec le grand réseau des routes anciennes de 'Uvea.

\section{Conclusion}

L'étude archéologique et la restauration de la plate-forme du Mālamatagata ont permis d'effectuer une analyse détaillée d'un monument traditionnel construit à 'Uvea au cours du deuxième millénaire après $\mathrm{J}$.-C. Il ne fait aucun doute que le site de 'Utuleve, occupé dès la période Lapita, durant toute la période céramique puis à la période tongienne, tient une place centrale dans nos connaissances sur l'histoire ancienne de 'Uvea (Frimigacci et Hardy,1994, 1995, 1996). Il est à souhaiter que d'autres recherches y soient menées prochainement afin de mieux comprendre les différentes étapes de l'implantation humaine sur cette île de la Polynésie occidentale.

\section{Remerciements}

Je remercie vivement Monsieur Claude Robineau pour ses suggestions et ses précisions qui nous ont permis d'améliorer cette contribution.

La rédaction du JSO remercie le CTRDP et les auteurs des photographies pour l'autorisation de reproduction des clichés 4 et 10 extraits de CTRDP (1995).

\section{BIBLIOGRAPHIE}

Burrows E. G., 1937, Ethnology of 'Uvea, Honolulu, Bernice P. Bishop Museum Bulletin 145.

FrimigaCCI Daniel et Maurice Hardy, 1994. Rapport d'activité, Programme de restauration et fouilles de sauvetage, service de la Sauvegarde du patrimoine culturel de Wallis-et-Futuna.

—, 1995. Rapport d'activité, Programme de restauration et fouilles de sauvetage, service de la Sauvegarde du patrimoine culturel de Wallis-et-Futuna.

—, 1996. Rapport d'activité, Programme de restauration et fouilles de sauvetage, service de la Sauvegarde du patrimoine culturel de Wallis-et-Futuna.

—, 1997. Des archéologues, des conquérants et des forts, Association socio-culturelle pour la culture et l'art wallisiens et futuniens, Versailles, Éditions Art Lys.

FrimigacCI Daniel, Jean-Pierre Siorat et Bernard VIENNE, 1982. Inventaire et fouille des sites archéologiques et ethnohistoriques de l'île de 'Uvea, documents provisoires, diffusion restreinte, Nouméa, centre ORSTOM.

_, 1995. Un poisson nommé uvea : éléments d'ethnohistoire de Wallis, Nouméa, CTRDP de NouvelleCalédonie, HIs 12.

Frimigacci Daniel et Bernard VIENNE, 2001. Wallis et Futuna, 3000 ans d'histoire, Nouméa, Association de la jeunesse wallisienne et futunienne en Nouvelle-Calédonie.

—, 2006. Les fondations du royaume de 'Uvea. Une histoire à revisiter, Journal de la Société des Océanistes 122-123 : Spécial Wallis-et-Futuna, pp. 27-60.

Henquel Joseph, 1910. Talanoa ki 'Uvea nei, Lano, Wallis, Presses de la Mission.

SAND Christophe, 1998. Uvéa, la préhistoire de Wallis, île de la Polynésie occidentale, Nouméa, édition Grain de sable. 